\section{El efecto de los microelementos sobre la germinación de semillas y el rendimiento de la masa verde de trigo de invierno en el periodo vegetativo}

Nikolay Myazin Georgievich ${ }^{1}$, Anna Kozhokina Nikolaevna 1 ( ), Petr Brekhov Timofevich'1 (), Tatiana Salgado Pacheco $0^{1,2}$

${ }^{1}$ Voronezh State Agrarian University named after Emperor Peter the Great (VSAU) 2Universidad Tecnológica de Panamá

1myazinnikolay@yandex.ru; 'annakozh27@yandex.ru; 'petr.brekhov@yandex.ru; 1,2tatiana.salgado@utp.ac.pa

DOI: 10.33412/pri.v12.1.2846

\section{다)(우(2)}

\section{Resumen}

Dado el crecimiento de la población en nuestro planeta, garantizar altos rendimientos de los cultivos se está convirtiendo en una tarea urgente. En la Federación Rusa, el principal cultivo alimenticio es el trigo de invierno, es imposible obtener altos rendimientos y de buena calidad sin la combinación óptima de macro y microelementos en el sistema de fertilización de este cultivo. En este sentido, se estudió el efecto del tratamiento previo a la siembra de semillas y hojas (no radiculares) con microelementos durante el crecimiento y desarrollo de la masa vegetativa del trigo de invierno. Se descubrió que el uso de microelementos para el tratamiento de semillas previo a la siembra, tanto con la composición de sales simples, como en preparaciones industriales (forma de quelato), se aumentó su germinación de campo en un 10.0-23.6\%, y se tuvo un efecto beneficioso sobre los parámetros biométricos de las plantas (la altura de la planta aumentó en $0.7-4.0 \mathrm{~cm}$, macollo en 0.1-0.3 unidades) y aumentó el rendimiento de la masa verde de trigo de invierno (en $0.3-2.1 \mathrm{~g} /$ recipiente). Al mismo tiempo, las plantas consumieron más nutrientes del suelo, como resultado de lo cual aumentó su contenido en la producción y la eliminación en la cosecha. La preparación industrial con Polydon Profi demostró la mayor efectividad en el experimento.

Palabras claves: trigo de invierno, microelementos, productividad, germinación de campo, eliminación de nutrientes.

Title: The effect of microelements on seed germination and the yield of the green mass of winter wheat in the vegetative period

Abstract: Given the population growth on our planet, ensuring high crop yields is becoming an urgent task. In the Russian
Federation, the main food crop is winter wheat, it is impossible to obtain high yields and of good quality without the optimal combination of macro and microelements in the fertilization system of this crop. In this sense, we study the effect of preplanting of seeds and leaves (non-root) with microelements during the growth and development of the vegetative mass of winter wheat. It was found that the use of microelements for seed treatment prior to sowing, both with the composition of simple salts, and in industrial preparations (chelate form), increased its field germination by $10.0-23.6 \%$, and it had a beneficial effect on the biometric parameters of the plants (the height of the plant increased by $0.7-4.0 \mathrm{~cm}$, tiller by $0.1-0.3$ units) and increased the yield of the green mass of winter wheat (by $0.3-2.1 \mathrm{~g} /$ container). At the same time, plants consumed more nutrients from the soil, as a result of which their content increased in production and removal at harvest. The industrial preparation with Polydon Profi demonstrated the highest effectiveness in the experiment.

Key words: winter wheat, microelements, productivity, field germination, nutrient removal.

Tipo de artículo: Estudio.

Fecha de recepción: 13 de agosto de 2020.

Fecha de aceptación: 5 de enero de 2021.

\section{Introducción}

Actualmente, el servicio de agroquímicos ha establecido que la mayoría de los suelos de la Federación de Rusia tienen un contenido insuficiente de microelementos. Por ejemplo, en la región de Vorónezh, el $99.7 \%$ de la superficie de tierra cultivable se caracteriza por un bajo suministro de zinc, $96.5 \%$ de cobre, $61 \%$ de manganeso [7]. Al mismo tiempo, las nuevas variedades de cultivos altamente productivos que tienen un tipo de metabolismo intensivo y se cultivan utilizando tecnologías intensivas, requieren un suministro suficiente de suelo con todos los nutrientes, incluidos los microelementos $[1,4,18]$. Por lo tanto, su déficit puede convertirse en un factor que restrinja el aumento del rendimiento y la calidad de los cultivos $[10,16]$.

La necesidad de fertilizantes con micronutrientes también está creciendo debido a la expansión del uso de fertilizantes con macronutrientes altamente concentrados, que se eliminan mejor y casi no contienen microelementos. Además, la introducción de mayores dosis de nitrógeno, fósforo y potasio desplaza el equilibrio iónico de la solución del suelo a menudo en la dirección desfavorable para la absorción de microelementos por las plantas $[2,3]$.

Mientras tanto, el uso de fertilizantes con micronutrientes no es tecnológicamente difícil y no requiere grandes gastos de mano de obra y dinero. Los costos de incrustación de semillas y tratamiento del material de siembra con fertilizantes con micronutrientes son en promedio 50 y $120 \mathrm{P}$ (rublos)/ha, respectivamente, y 200P(rublos)/ha para el abono foliar, con este método se asegura la recepción de $3,14 \mathrm{P}$ (rublos) de ingresos netos por cada rublo gastado $[5,6,14]$.

En la actualidad ha habido una tendencia a usar fertilizantes de micronutrientes quelados con una acción más efectivo en 
comparación con las sales simples, sin embargo, las tecnologías para su aplicación requieren un estudio más detallado en las condiciones específicas de la producción agrícola $[13,17]$. Uno de los principales cultivos es el trigo de invierno. Según Rosstat en 2018, representó el $56,1 \%$ de todos los cultivos en la Federación de Rusia. El rendimiento promedio anual de la cosecha no fue alto y ascendió a $35.1 \mathrm{q} /$ ha [15]. El impulso para aumentar su productividad puede ser la introducción de tecnologías avanzadas en el proceso de cultivo de trigo de invierno, incluido el uso de nuevos tipos de fertilizantes con micronutrientes en forma de quelato $[8,9,19]$.

Al respecto, el propósito de la presente investigación fue establecer el efecto del tratamiento de semillas y fertilización foliar con microelementos que se encuentran en la composición de fertilizantes tanto en forma de sales simples, como en forma quelada para la germinación de semillas en el campo, desarrollo vegetal y rendimiento de masa verde de trigo de invierno en las condiciones de un experimento durante el periodo vegetativo.

\section{Materiales y métodos}

La evaluación de la acción de microelementos sobre el trigo de invierno se llevó a cabo en un experimento de crecimiento. Se estudió la variedad de trigo de invierno "Scarlet Dawn" de cría en FSBEI HE Voronezh State Agrarian University. Los fertilizantes utilizados en el experimento fueron: nitrato de amonio, urea, cloruro de potasio, superfosfato doble, azofoska. Se usaron microelementos (cobre, zinc, manganeso, boro, etc.) como parte de compuestos simples ( $\mathrm{CuSO}_{4}, \mathrm{ZnSO}_{4}, \mathrm{MnSO}_{4}, \mathrm{H}_{3} \mathrm{BO}_{3}$ ), y como parte de complejos, con Trilon $\mathrm{B}$ y varios otros agentes complejantes, presentados en preparaciones industriales "Polydon Amino Start" y "Polydon Profi". La composición y las propiedades de los compuestos utilizados se presentan en la tabla 1.

Tabla 1. Composición y propiedades de las preparaciones "Polydon Amino Start" y "Polydon Profi".

\begin{tabular}{|l|c|c|}
\hline \multirow{2}{*}{\multicolumn{1}{|c|}{ Indicadores }} & \multicolumn{2}{c|}{ Valores } \\
\cline { 2 - 3 } & $\begin{array}{c}\text { Polydon Amino } \\
\text { Start }\end{array}$ & Polydon Profi \\
\hline L * aminoácidos y oligopéptidos & $250 \mathrm{~g} / \mathrm{L}$ & - \\
\hline Sustancias húmicas (ON) & - & $10.0 \mathrm{~g} / \mathrm{L}$ \\
\hline Aminoácidos (L-AA) & - & $20 \mathrm{~g} / \mathrm{L}$ \\
\hline Nitrógeno (N total) & $100 \mathrm{~g} / \mathrm{L}$ & $50.0 \mathrm{~g} / \mathrm{L}$ \\
\hline Fósforo (P2O5) & $75 \mathrm{~g} / \mathrm{L}$ & $10.0 \mathrm{~g} / \mathrm{L}$ \\
\hline Potasio (K2O) & $25 \mathrm{~g} / \mathrm{L}$ & $10.0 \mathrm{~g} / \mathrm{L}$ \\
\hline Calcio (CaO) & - & $1.0 \mathrm{~g} / \mathrm{L}$ \\
\hline Azufre (SO3) & - & $10.0 \mathrm{~g} / \mathrm{L}$ \\
\hline Magnesio (MgO) & $15 \mathrm{~g} / \mathrm{L}$ & $3.0 \mathrm{~g} / \mathrm{L}$ \\
\hline Sodio (Na2O) & - & $1.0 \mathrm{~g} / \mathrm{L}$ \\
\hline Silicio (SiO2) & - & $0.5 \mathrm{~g} / \mathrm{L}$ \\
\hline Hierro (Fe) & $6 \mathrm{~g} / \mathrm{L}$ & $0.75 \mathrm{~g} / \mathrm{L}$ \\
\hline Manganeso (Mn) & $3 \mathrm{~g} / \mathrm{L}$ & $0.375 \mathrm{~g} / \mathrm{L}$ \\
\hline Zinc (Zn) & $3 \mathrm{~g} / \mathrm{L}$ & $0.15 \mathrm{~g} / \mathrm{L}$ \\
\hline
\end{tabular}

\begin{tabular}{|l|c|c|}
\hline Cobre $(\mathrm{Cu})$ & $3 \mathrm{~g} / \mathrm{L}$ & $0.15 \mathrm{~g} / \mathrm{L}$ \\
\hline Boro $(\mathrm{B})$ & $3 \mathrm{~g} / \mathrm{L}$ & $0.1 \mathrm{~g} / \mathrm{L}$ \\
\hline Molibdeno $(\mathrm{Mo})$ & $1 \mathrm{~g} / \mathrm{L}$ & $0.5 \mathrm{~g} / \mathrm{L}$ \\
\hline Cobalto $(\mathrm{Co})$ & $0.05 \mathrm{~g} / \mathrm{L}$ & $0.015 \mathrm{~g} / \mathrm{L}$ \\
\hline $\mathrm{pH}$ & 3.8 & $8.5-8.7$ \\
\hline Densidad & $1.245 \mathrm{~g} / \mathrm{cm}^{3}$ & $1.15 \mathrm{~g} / \mathrm{cm}^{3}$ \\
\hline
\end{tabular}

El experimento en la etapa vegetativa se realizó el 19 de abril de 2019 de acuerdo con el esquema que se muestra en la tabla 3 . En el experimento, se utilizaron recipientes cilíndricos con un diámetro de $20 \mathrm{~cm}$ y una altura de $40 \mathrm{~cm}$. La masa de tierra seca fue de $10 \mathrm{~kg}$. El suelo en los vasos (a excepción de la versión de control) se mezcló previamente con macroelementos en una dosis de $\mathrm{N}_{0.1} \mathrm{P}_{0.1} \mathrm{~K}_{0.1}$ (g / kg de suelo absolutamente seco), al mismo tiempo, el recipiente contenía $6.25 \mathrm{~g}$ de nitrofoska (marca 16:16:16). La repetición de la experiencia se realizó por quintuplicado.

Las propiedades agroquímicas del suelo utilizado para el experimento de cultivo se presentan en la tabla 2. Antes de iniciar la experiencia, el suelo se clasificó según el grado de acidez como ácido medio y contenía demás un alto grado de saturación con bases.

El contenido de fósforo móvil corresponde a la clase media y el intercambio de potasio elevado.

Tabla 2. Propiedades agroquímicas del suelo antes de iniciar la experiencia.

\begin{tabular}{|c|c|c|c|c|c|c|}
\hline \multirow[t]{2}{*}{ pH KcI } & $\begin{array}{l}\text { Acidez } \\
\text { hidrolitica } \\
\text { (Hh) }\end{array}$ & s & $\mathrm{T}$ & \multirow[t]{2}{*}{$\mathrm{V}, \%$} & \multicolumn{2}{|c|}{$\begin{array}{l}\text { Contenido de formas } \\
\text { móviles, } \\
\mathrm{mg} / \mathrm{kg} \text { de suelo (según } \\
\text { Chirikov) }\end{array}$} \\
\hline & \multicolumn{3}{|c|}{ meq/100 g suelo } & & $\mathrm{P}_{2} \mathrm{O}_{5}$ & $\mathrm{~K}_{2} \mathrm{O}$ \\
\hline 4.8 & 4.6 & 25.4 & 30.0 & 84.7 & 68 & 113 \\
\hline
\end{tabular}

Para el tratamiento previo a la siembra de semillas y la alimentación foliar de plantas de trigo con microelementos, se utilizaron soluciones al $0.05 \% \mathrm{CuSO}_{4}, \mathrm{ZnSO}_{4}, \mathrm{MnSO}_{4}$ и $\mathrm{H}_{3} \mathrm{BO}_{3}$. La solución de trabajo "Polydon Amino Start" se preparó de acuerdo con las instrucciones de uso por dilución en agua veinte veces. La solución de trabajo "Polydon Profi" - dilución diez veces.

Los granos de trigo de invierno se trataron con soluciones de trabajo con una exposición de diez segundos, esto garantizaba el contacto total de toda la superficie de los granos con la preparación, y de igual forma se aproximaba más a los efectos de la solución a través del tiempo en la tecnología de producción con tratamiento previo a la siembra de semillas de cultivos.

El riego de las plantas durante el período de crecimiento se realizó no con agua natural, sino con agua destilada (para excluir el efecto distorsionador de las sales contenidas en el agua natural).

La alimentación foliar de trigo de invierno con microelementos se llevó a cabo en el medio y al final de la fase de macollaje. La cosecha de la masa verde de las plantas de trigo de invierno se registró el 06.06.2019.

El análisis agroquímico de muestras de suelo y plantas se realizó en el laboratorio del Departamento de Agroquímica, 
Ciencia del Suelo y Agroecología utilizando métodos generalmente aceptados [11].

\section{Resultados y discusión}

Bajo las condiciones de la temporada de crecimiento, se estudió el efecto complejo de los microelementos sobre el trigo de invierno mediante el tratamiento previo a la siembra y el tratamiento de las hojas. Las condiciones del suelo con el cual se realizó la investigación y sus parámetros fisicoquímicas se encuentran enlistados en la (tabla 3).

En relación con el control (sin fertilizantes), se observó acidificación del suelo en todas las variantes estudiadas, sin embargo, la relación entre las diferentes variantes con microelementos es insignificante.

Tabla 3. Propiedades fisicoquímicas de chernozem lixiviado (cosecha de masa verde de trigo de invierno).

\begin{tabular}{|l|c|c|c|c|c|c|}
\hline \multirow{2}{*}{ Tratamientos } & \multirow{2}{*}{$\mathrm{pH}_{\mathrm{KCl}}$} & $\mathrm{Hh}$ & $\mathrm{S}$ & $\mathrm{T}$ & \multirow{2}{*}{$\mathrm{V}, \%$} \\
\cline { 3 - 5 } & & 4.8 & 4.6 & 25.4 & 30.0 & 84.7 \\
\hline 1. Sin fertilizantes - control & 4.6 & 4.9 & 25.2 & 30.1 & 83.7 \\
\hline 2. $\mathrm{N}_{0.1} \mathrm{P}_{0.1} \mathrm{~K}_{0.1}$ - Fondo & 4.6 & 4.8 & 25.7 & 30.5 & 84.3 \\
\hline $\begin{array}{l}\text { 3. Fondo + Polydon Amino } \\
\text { Start }\end{array}$ & 4.6 & 5.0 & 25.5 & 30.5 & 83.6 \\
\hline 4. Fondo + Polydon Profi & 4.6 & 4.9 & 25.7 & 30.6 & 84.0 \\
\hline $\begin{array}{l}\text { 5. Fondo + } \\
\text { microelementos + Trilon B }\end{array}$ & 4.6 & 4.9 & 25.2 & 30.1 & 83.7 \\
\hline $\begin{array}{l}\text { 6. Fondo + } \\
\text { microelementos + } \mathrm{HNO}_{3}\end{array}$ & & & & \\
\hline
\end{tabular}

Se encontró el mismo comportamiento sobre el contenido de nutrientes en el suelo de fondo y las variantes con fertilizantes con micronutrientes (tabla 4).

Tabla 4. El contenido de nutrientes en el suelo al cosechar masa verde de trigo de invierno, $\mathrm{mg} / \mathrm{kg}$ de suelo.

\begin{tabular}{|c|c|c|c|c|c|}
\hline $\begin{array}{c}\text { Variante } \\
\text { experimental }\end{array}$ & $\mathrm{N}-\mathrm{NO}_{3}$ & $\mathrm{~N}-\mathrm{NH}_{4}$ & $\sum_{\substack{\left.\mathrm{N}-\mathrm{NO}_{3}+\mathrm{N}-\\
\mathrm{NH}_{4}\right)}}$ & $\mathrm{P}_{2} \mathrm{O}_{5}$ & $\mathrm{~K}_{2} \mathrm{O}$ \\
\hline $\begin{array}{l}\text { 1. Sin } \\
\text { fertilizantes - } \\
\text { control }\end{array}$ & 1.9 & 2.0 & 3.9 & 60 & 97 \\
\hline $\begin{array}{l}\text { 2. } \mathrm{N}_{0.1} \mathrm{P}_{0.1} \mathrm{~K}_{0.1} \text { - } \\
\text { Fondo }\end{array}$ & 4.8 & 3.0 & 7.8 & 100 & 97 \\
\hline $\begin{array}{l}\text { 3. Fondo + } \\
\text { Polydon Amino } \\
\text { Start }\end{array}$ & 2.9 & 3.3 & 6.2 & 100 & 96 \\
\hline $\begin{array}{l}\text { 4. Fondo + } \\
\text { Polydon Profi }\end{array}$ & 3.1 & 3.5 & 6.6 & 100 & 95 \\
\hline $\begin{array}{l}\text { 5. Fondo + } \\
\text { microelemento } \\
\mathrm{s}+\text { Trilon B }\end{array}$ & 4.0 & 3.4 & 7.4 & 100 & 96 \\
\hline $\begin{array}{l}\text { 6. Fondo + } \\
\text { microelemento } \\
\mathrm{s}+\mathrm{HNO}_{3}\end{array}$ & 4.7 & 2.0 & 6.7 & 100 & 96 \\
\hline
\end{tabular}

El contenido de fósforo en todas estas variantes fue de 100 $\mathrm{mg} / \mathrm{kg}$ de suelo, y el contenido de potasio disminuyó ligeramente (de 1 a $2 \mathrm{mg} / \mathrm{kg}$ de suelo). La disminución en el contenido de nitrógeno mineral en relación con el fondo fue casi la misma, entre 0.4-1.6 $\mathrm{mg} / \mathrm{kg}$ de suelo.
Los macro fertilizantes introducidos en el suelo y el tratamiento previo a la siembra de semillas con soluciones que contienen microelementos influyó de manera diferente en el crecimiento y desarrollo del trigo de invierno. La germinación de campo de las semillas en las variantes de control y de fondo no fue muy diferente (figura 1).

El tratamiento de semillas con sales simples de microelementos en combinación con un agente complejante (Trilon B, opción 5) aumentó la germinación de campo en 6.0 puntos porcentuales, y en combinación con ácido nítrico, en 11.1 puntos porcentuales en relación con el fondo.

Germinación en campo de semillas (\%)

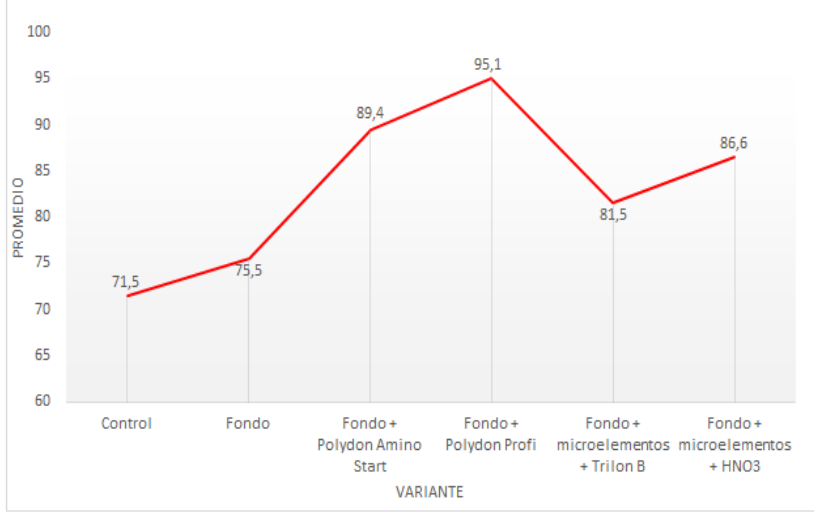

Figura 1. El efecto de los fertilizantes en la germinación de semillas en el campo (\%).

El mayor aumento en la germinación en el campo se observó en el caso del tratamiento previo a la siembra de semillas con las preparaciones Polydon Amino Start y, en particular, con la preparación Polydon Profi (en 13,9 y 19,6 puntos porcentuales, respectivamente).

Así, el uso de micronutrientes para el tratamiento previo de semillas, tanto en la composición de sales simples como en forma de preparaciones industriales, aumentó su germinación en el campo. El mayor impacto positivo en este indicador fue proporcionado por preparaciones complejas, especialmente Polydon Profi. Vale la pena señalar qué al estudiar el efecto de estas preparaciones sobre la energía de germinación y la germinación de las semillas en el laboratorio, se obtuvieron casi los mismos patrones [12].

El uso de fertilizantes contribuyó a la creación de condiciones más favorables para el desarrollo de las plantas en el período posterior de su vegetación. Por lo tanto, se observó el macollamiento más pequeño de las plantas en el experimento (1.0 unidades) en la variante de control (Figura 2). El uso de fertilizantes que contienen solo macronutrientes (opción 2) lo aumentó en 1.9 unidades. $Y$ el tratamiento de semillas con micronutrientes en combinación con el apósito foliar (variantes 36) aumentó en 2.0-2.2 unidades. en comparación con el control. Al mismo tiempo, se observó el mayor macollaje del trigo de invierno cuando se usó la preparación Polydon Profi. Entre la 
preparación "Polydon Amino Start" y las sales de microelementos utilizadas junto con Trilon B y ácido nítrico, prácticamente no hubo diferencias en este indicador.

Similares patrones se observan con respecto a la altura de las plantas en el experimento. La única diferencia que se encontró como efecto positivo de la preparación de Polydon Amino Start fue una presentación ligeramente más brillante en comparación con las formas minerales de microelementos (variante 5 y 6 ). El aumento de altura de la planta, así como su macollaje, se observó únicamente en la variante que usa la preparación Polydon Profi.

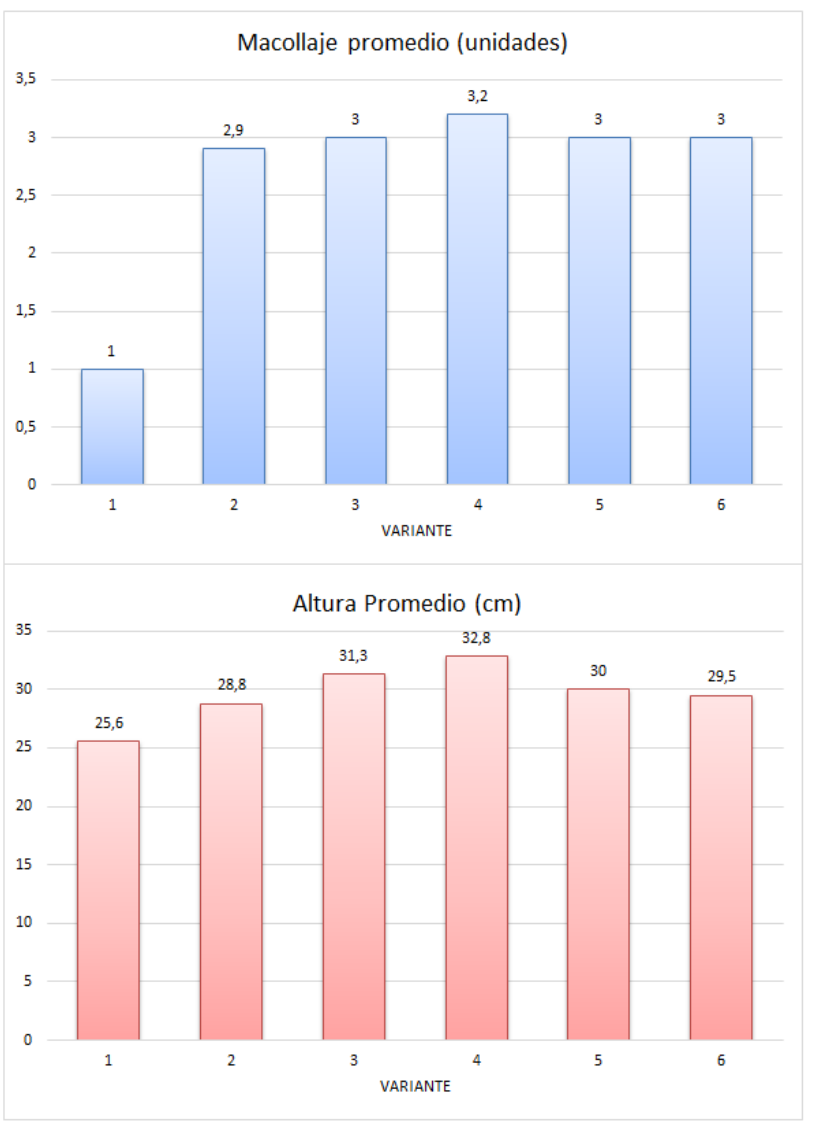

Figura 2. El efecto de los fertilizantes sobre la maleza y la altura de la planta.

Variantes experimentales: 1. Control 2. Fondo; 3. Fondo + Polydon Amino Start; 4. Fondo +Polydon Profi; 5. Fondo + microelementos + Trilon $\mathrm{B} ; \mathbf{6}$. Fondo + microelementos $+\mathrm{NO}_{3}$

Tomando en cuenta la cosecha de masa verde de trigo de invierno en el experimento (tabla 5) se demostró que la aplicación de fertilizantes minerales de fondo estuvo acompañada por un fuerte aumento en el rendimiento de la masa verde de trigo, en un $241 \%$.
Tabla 5. El rendimiento de la masa verde del trigo de invierno.

\begin{tabular}{|c|c|c|c|}
\hline Variante experimental & $\begin{array}{c}\text { Peso de } \\
\text { masa } \\
\text { verde }\end{array}$ & $\begin{array}{l}\text { Incremento } \\
\text { en el control }\end{array}$ & $\begin{array}{l}\text { Incremento } \\
\text { en el Fondo }\end{array}$ \\
\hline & \multicolumn{3}{|c|}{ g/recipiente } \\
\hline $\begin{array}{l}\text { 1. Sin fertilizante - } \\
\text { Control }\end{array}$ & 13.9 & - & - \\
\hline 2. $\mathrm{N}_{0.1} \mathrm{P}_{0.1} \mathrm{~K}_{0.1}$ - Fondo & 47.4 & 33.5 & - \\
\hline $\begin{array}{l}\text { 3. Fondo + Polydon } \\
\text { Amino Start }\end{array}$ & 48.1 & 34.2 & 0.7 \\
\hline 4. Fondo +Polydon Profi & 49.5 & 35.6 & 2.1 \\
\hline $\begin{array}{l}\text { 5. Fondo + } \\
\text { microelementos + Trilon }\end{array}$ & 47.9 & 34.0 & 0.5 \\
\hline $\begin{array}{l}\text { 6. Fondo }+ \\
\text { microelementos }+\mathrm{HNO}_{3}\end{array}$ & 47.7 & 33.8 & 0.3 \\
\hline $\mathrm{HCP}_{0.95}$, г/сосуд & 1.90 & - & - \\
\hline$S_{x}, \%$ & 1.51 & - & - \\
\hline
\end{tabular}

El uso de microelementos para el tratamiento previo a la siembra de semillas y el recubrimiento foliar de plantas en el contexto de la aplicación de $\mathrm{N}_{0.1} \mathrm{P}_{0.1} \mathrm{~K}_{0.1}$ al suelo proporcionó un aumento adicional en el rendimiento de la masa verde de trigo de invierno en 0.3-2.1 g/recipiente en comparación con la opción 2. En este caso, el aumento demostrable matemáticamente en el rendimiento para el fondo fue proporcionado solo por el uso de la preparación Polydon Profi (opción 4). Vale la pena señalar que el estado de las plantas en esta variante fue notablemente mejor antes de la cosecha que en otras variantes del experimento (figura 3). 


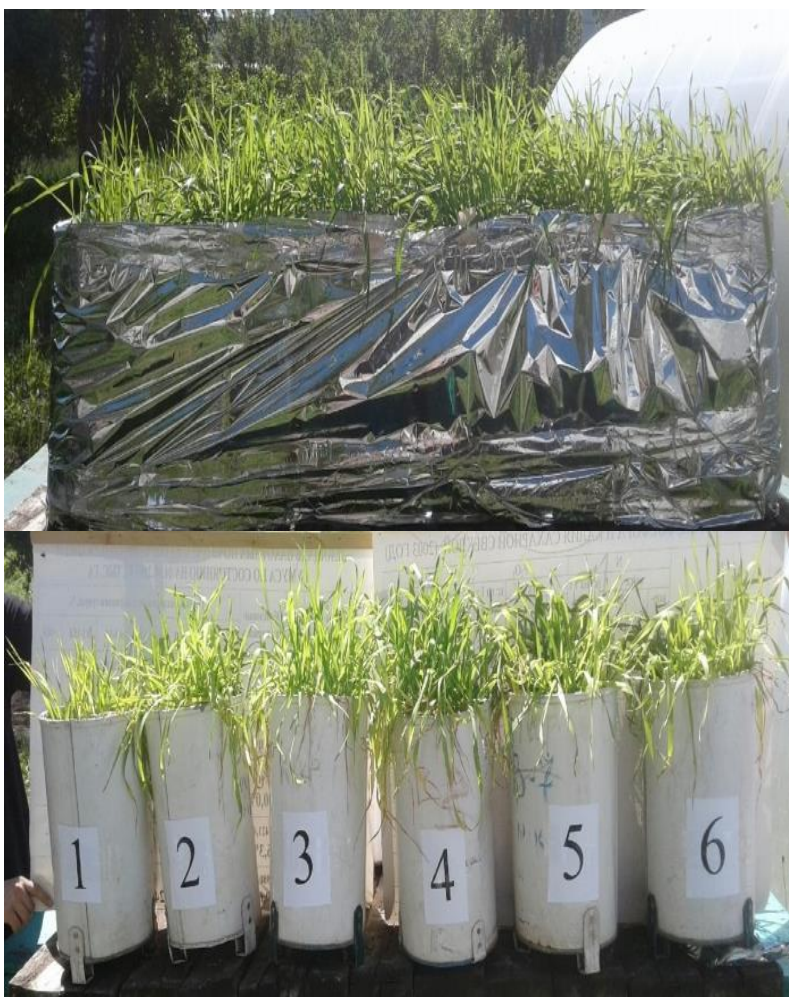

Figura 3. Condición de las plantas de trigo de invierno antes de la cosecha durante la experiencia vegetativa.

Variantes experimentales: 1. Control 2. Fondo; 3. Fondo + Polydon Amino Start; 4. Fondo +Polydon Profi; 5. Fondo + microelementos + Trilon $\mathrm{B} ; 6$. Fondo + microelementos $+\mathrm{NO}_{3}$

Las variantes que utilizaron microelementos diferían entre sí, sin embargo, matemáticamente estas diferencias no eran demostrables.

Solo se puede hablar de una cierta tendencia de mejor acción (en orden decreciente) de los preparativos Polydon Profi y Polydon Amino Start (variantes 3 y 4), comparados con la aplicación de soluciones tradicionales de microelementos (variantes 5 y 6 ), al mismo tiempo, la efectividad de las variantes 5 y 6 , donde se utilizaron microelementos en forma de sales complejas y simples respectivamente, no presentaron una diferencia significativa.

El contenido de nutrientes en la masa seca del trigo de invierno (tabla 6) también dependía de la aplicación de fertilizantes. En la versión no fertilizada, fue mínima en el experimento.

El uso de fertilizantes, aumentando el contenido de nutrientes en el suelo, creó las condiciones para su mayor consumo por parte de las plantas, por lo tanto, el contenido de nitrógeno en la masa seca del trigo de invierno desde la introducción de azofoska (variante 2) aumentó en 2.33 puntos porcentuales, y cuando se combina con azofoska y soluciones que contienen oligoelementos, en 2.4 - 2.86 puntos porcentuales. Patrones similares obedecieron al cambio en el contenido de fósforo y potasio en las plantas.
Tabla 6. Contenido en porcentaje de NPK en peso seco de trigo de invierno.

\begin{tabular}{|l|c|c|c|}
\hline \multicolumn{1}{|c|}{ Variante experimental } & $\mathbf{N}$ & $\mathbf{P}_{2} \mathbf{O}_{5}$ & $\mathbf{K}_{2} \mathbf{O}$ \\
\hline 1. Sin fertilizante - Control & 1.46 & 1.13 & 3.38 \\
\hline 2. No.1P0.1 K.1 - Fondo & 3.79 & 1.79 & 5.34 \\
\hline $\begin{array}{l}\text { 3. Fondo + Polydon Amino } \\
\text { Start }\end{array}$ & 3.86 & 1.84 & 5.40 \\
\hline 4. Fondo + Polydon Profi & 4.10 & 1.98 & 5.46 \\
\hline $\begin{array}{l}\text { 5. Fondo + microelementos } \\
\text { + Trilon }\end{array}$ & 4.32 & 2.05 & 5.56 \\
\hline $\begin{array}{l}\text { 6. Fondo + microelementos } \\
+\mathrm{HNO}_{3}\end{array}$ & 3.86 & 1.87 & 5.30 \\
\hline
\end{tabular}

La fertilización foliar en trigo de invierno aumentó el contenido en la masa verde de las plantas, todos los microelementos introducidos, y especialmente el cobre y el manganeso (tabla 7). aumentaron, respectivamente, de 2.43 y $140 \mathrm{mg} / \mathrm{g}$ a 3.16 y $180 \mathrm{mg} / \mathrm{kg}$ de masa seca de plantas sobre el suelo, es decir, aproximadamente 1.3 veces.

Tabla 7. El contenido de oligoelementos en la masa verde del trigo de invierno ( $\mathrm{mg} / \mathrm{kg}$ ), el final del macollamiento.

\begin{tabular}{|l|l|l|l|l|}
\hline \multicolumn{1}{|c|}{ Variante experimental } & $\mathrm{Cu}$ & $\mathrm{Zn}$ & $\mathrm{Mn}$ & $\mathrm{B}$ \\
\hline 1. Sin fertilizante - Control & 2.25 & 25.3 & 115 & 5.1 \\
\hline 2. No.1Po.1Ko.1 - Fondo & 2.43 & 65.9 & 140 & 6.2 \\
\hline $\begin{array}{l}\text { 3. Fondo + Polydon Amino } \\
\text { Start }\end{array}$ & 2.99 & 70.4 & 180 & 6.4 \\
\hline 4. Fondo +Polydon Profi & 3.16 & 68.6 & 182 & 6.7 \\
\hline $\begin{array}{l}\text { 5. Fondo + microelementos } \\
\text { + Trilon }\end{array}$ & 2.98 & 67.8 & 178 & 6.6 \\
\hline $\begin{array}{l}\text { 6. Fondo + microelementos } \\
\text { + } \mathrm{HNO}_{3}\end{array}$ & 2.99 & 68.5 & 180 & 6.5 \\
\hline Contenido óptimo & $\begin{array}{l}5.00- \\
10.00\end{array}$ & $\begin{array}{l}20.0- \\
70.0\end{array}$ & $\begin{array}{l}90- \\
134\end{array}$ & $\begin{array}{l}2.0- \\
8.0\end{array}$ \\
\hline
\end{tabular}

Se puede observar que el contenido de zinc y boro corresponde a niveles óptimos, y el contenido de manganeso a un nivel alto, únicamente el contenido de cobre permanece por debajo del nivel óptimo.

Cabe resaltar que en el experimento el mayor aumento en el contenido de cobre, manganeso y boro en las plantas es proporcionado por la preparación Polydon Profi. Y el mayor aumento en zinc se observó a partir de la acción de la preparación "Polydon Amino Start".

Por lo tanto, el efecto combinado de las soluciones de micronutrientes (en las semillas antes de la siembra y en las hojas de las plantas en el futuro) aumenta el contenido de todos los microelementos en la masa verde del trigo de invierno y les proporciona a todos niveles óptimos y altos en las plantas, con excepción del cobre cuyo contenido en la masa verde también aumenta notablemente, sin embargo, este no alcanza el óptimo.

El cálculo de la eliminación de nutrientes de las plantas (tabla 8) se observó que era mínima en la variante de control, la aplicación de fertilizante que contiene únicamente macroelementos (variante 2) aumentó la eliminación en 0.30, 0.13 y $0.38 \mathrm{~g} /$ recipiente, respectivamente, para nitrógeno, fósforo y potasio en relación con la variante de control, cuando se utilizan 
microelementos, la eliminación de nitrógeno aumentó en el fondo en 10-50mg/recipiente, la eliminación de fósforo en 10 $30 \mathrm{mg} /$ recipiente y el potasio también en 10-30 mg/recipiente.

Las variantes experimentales con microelementos proporcionaron un aumento en las tasas de utilización de nutrientes fertilizantes en un $3.0-16.0 \%$ para el nitrógeno, $7.0-$ $23 \%$ para el fósforo y $3.0-8.0 \%$ para el potasio en relación con la variante de fondo. El mayor aumento en la utilización de nutrientes fertilizantes se observó con la variante Polydon Profi (16.7\% para nitrógeno, $23.1 \%$ para fósforo y $7.9 \%$ para potasio) las otras variantes con microelementos para este indicador, especialmente en relación con el fósforo y el potasio no diferían.

Tabla 8. Eliminación de nutrientes y coeficiente de utilización de reservas de suelo y fertilizantes del trigo de invierno.

\begin{tabular}{|l|c|l|l|l|l|l|}
\hline \multirow{2}{*}{$\begin{array}{c}\text { Variante } \\
\text { experimental }\end{array}$} & \multicolumn{3}{|c|}{$\begin{array}{c}\text { Eliminación de } \\
\text { nutrientes } \\
\text { (g/recipiente) }\end{array}$} & \multicolumn{3}{c|}{$\begin{array}{c}\text { Coeficiente de } \\
\text { utilización de } \\
\text { fertilizantes (\%) }\end{array}$} \\
\cline { 2 - 7 } & $\mathrm{N}$ & $\mathrm{P}_{2} \mathrm{O}_{5}$ & $\mathrm{~K}_{2} \mathrm{O}$ & $\mathrm{N}$ & $\mathrm{P}_{2} \mathrm{O}_{5}$ & $\mathrm{~K}_{2} \mathrm{O}$ \\
\hline $\begin{array}{l}\text { 1. Sin fertilizante - } \\
\text { Control }\end{array}$ & 0.06 & 0.04 & 0.13 & - & $8.2^{*}$ & $15.1^{*}$ \\
\hline $\begin{array}{l}\text { 2. No.1P0.1 } \mathrm{K}_{0.1} \text { - } \\
\text { Fondo }\end{array}$ & 0.36 & 0.17 & 0.51 & 30 & 13 & 38 \\
\hline $\begin{array}{l}\text { 3. Fondo + } \\
\text { Polydon Amino } \\
\text { Start }\end{array}$ & 0.37 & 0.18 & 0.52 & 31 & 14 & 39 \\
\hline $\begin{array}{l}\text { 4. Fondo } \\
\text { +Polydon Profi }\end{array}$ & 0.41 & 0.20 & 0.54 & 35 & 16 & 41 \\
\hline $\begin{array}{l}\text { 5. Fondo + } \\
\text { microelementos + } \\
\text { Trilon }\end{array}$ & 0.39 & 0.18 & 0.50 & 33 & 14 & 37 \\
\hline $\begin{array}{l}\text { 6. Fondo + } \\
\text { microelementos + } \\
\text { HNO } 3\end{array}$ & 0.38 & 0.18 & 0.52 & 32 & 14 & 39 \\
\hline $\begin{array}{l}\text { * para la variante de control, se dan los coeficientes de uso } \\
\text { de nutrientes de las reservas del suelo }\end{array}$ \\
\hline
\end{tabular}

\section{Conclusión}

En el experimento de crecimiento, los estudios han demostrado que el uso de microelementos aumenta la germinación en el campo de las semillas de trigo de invierno (en un 10-23,6\%), aumenta el arbusto o macollo de las plantas en 0,10,3 unidades, la longitud de las hojas (en $0.7-4.0 \mathrm{~cm}$ ), el rendimiento de la masa verde (en $0.3-2.1 \mathrm{~g} /$ recipiente) y su calidad, y también aumenta la utilización de nutrientes de los fertilizantes - en nitrógeno en 3.3-16.7\%, en fósforo en 7.7-23.1 $\%$, potasio en $2.6-7.9 \%$, en comparación con el uso de fertilizantes que contienen solo macronutrientes.

La más efectiva en el experimento fue la variante con la preparación Polydon Profi, por debajo de este encontramos la preparación Polydon Amino Start. El uso de microelementos juntos con trilon $B$ (forma de quelato) y en forma de sales simples tuvieron el mismo efecto, el cual a su vez fue menor al de las preparaciones industriales.

\section{Referencias}

[1] A.M. Zhabin [et al.], Evaluación agroecológica de suelos de granjas en el sureste de la región de Voronezh, Boletín Agroquímico No. 2, pág. 8-9, Voronezh, Rusia, 2004.

[2] P.I. Anspock, Microfertilizantes, Ed. Handbook / Agropromizdat, 272 pág., Rusia, 1990.

[3] P.A. Vlasyuk, Microelementos en el medio ambiente, ed. Naukova Dumka Kiev, Ucrania, 247 pág., 1980.

[4] V. B. llyin, Biogeoquímica y agroquímica de oligoelementos ( $\mathrm{Mn}, \mathrm{Cu}, \mathrm{Mo}, \mathrm{B})$ en la parte sur de Siberia occidental, Ed. Nauka, Novosibirsk, Rusia: 390 pág., 1973.

[5] A. Kabata-Pendias, Microelementos en suelos y plantas, ed. Mundo, Moscú, Rusia, 439 pág., 1989.

[6] M.V. Katalymov, Microelementos y su papel en el aumento de la productividad, Ed. Goskhimizdat, Moscú, Rusia, 64 pág., 1957.

[7] V.I. Korchagin, Evaluación ecológica y agroquímica de la fertilidad del suelo en la región de Voronezh, 256 pág., Ed. Nauka, Voronezh, Rusia, 2017.

[8] V.I. Kostin, F.A. Mudarisov, A.I. Semashkina, Influencia de los microelementos: sinergistas sobre los parámetros fotosintéticos y el rendimiento del trigo de invierno, Boletín de la Academia Agrícola del Estado de Uliánovsk, no 4 (40). pág. 30-35, Uliánovsk, Rusia, - 2017.

[9] V.I. Lazarev, A.B. Vartanova, La influencia de los fertilizantes complejos con microelementos en el rendimiento y la calidad del grano de trigo de invierno en la región de Kursk, Boletín de la Academia Agrícola del Estado de Kursk. No. 6B, pág. 45-48, Kursk, Rusia 2014.

[10] I.A. Lebedovsky, I.V. Shabanova, E.A. Yakovleva, Influencia de microelementos en la productividad y calidad del trigo de invierno cultivado en chernozem lixiviado de la Ciscaucasia Occidental, Revista científica electrónica matemática política de la Universidad Agraria del Estado de Kuban, № 82. pág. 684-695, Kuban, Rusia, 2012

[11] V.G. Mineev, V.G. Sychev, O.A. Amelyanchik [et al.], Taller sobre Agroquímica, Ed. Universidad Estatal de Moscú, 689 pág., Moscú, Rusia 2001

[12] N.G. Myazin, P.T. Brekhov, A.N. Kozhokina, Influencia del tratamiento previo a la siembra de semillas de trigo de invierno con microelementos en energía de germinación y germinación de laboratorio / Biologización agrícola: perspectivas y posibilidades reales Materiales de la conferencia internacional científico-práctica dedicada al 105 aniversario del Doctor en Ciencias Agrícolas, Profesor, Miembro correspondiente de VASKHNIL Mikhail Ivanovich Sidorov y el 70 cumpleaños del Doctor en Ciencias Agrícolas, Profesor Nikolai Ivanovich Zezyukov, Ed. FSBEI Universidad Estatal Agraria de Voronezh, pág. 322-328. Vorónezh, Rusia 2019

[13] Yu.M. Isaev [et al.], Estudio experimental del efecto de oligoelementos en el rendimiento del trigo de invierno, International Journal of Experimental Education. No. 10-2, pág. 236, Rusia, 2016

[14] Ya.V. Peive, Pautas para el uso de microfertilizantes, Ed. Selkhozizdat, 224 pág., Rusia, 1963

[15] Área sembrada, cosecha bruta y rendimiento de trigo en Rusia. Resultados de 2018. https://agrovesti.net/lib/industries/cereals/posevnye-ploshchadi -valovye-sbory-i-urozhajnost-pshenitsy-v-rossii-itogi-2018-goda.html

[16] N.A. Protasova, A.P. Shcherbakov, Microelementos (Cr, V, Ni, Mn, Zn, Cu, $\mathrm{Co}, \mathrm{Ti}, \mathrm{Zr}, \mathrm{Ga}, \mathrm{Be}, \mathrm{Sr}, \mathrm{Ba}, \mathrm{B}, \mathrm{I}, \mathrm{Mo}$ ) en chernozems y suelos de bosque gris de la región de Chernozem Central, Ed. FGBOU VO Universidad Estatal de Voronezh, pág. 368, Voronezh, Rusia, 2003.

[17] Yu.I. Stolpovsky, Microelementos y Microfertilizantes, Ed. FGBOU VPO Voronezh SAU, pág. 171, Voronezh, Rusia, 2015.

[18] D.E. Ulanova, Yu.I. Zhitin, N.V. Stekolnikov, La influencia de la introducción de residuos de la industria alimentaria en el funcionamiento de los agrocenosos en la Región Central de Chernozem, Boletín de la Universidad Estatal Agraria de Voronezh. Núm. 4 (47), pág. 27-33, Vorónezh, Rusia, 2015.

[19] V.B. Shchukin, A.A. Gromov, N.V. Shchukina, Influencia del apósito foliar con microelementos en la formación del tallo productivo por las plantas de trigo de invierno, Noticias de la Universidad Agraria Estatal de Orenburg No. 3 (11), pág. 10-12, Oremburgo, Rusia, 2006. 